\title{
Zooming in on Cell Architecture and Molecular Structures with Correlative Light and Electron Microscopy
}

Maarten W. Tuijtel, Charlotte E. Melia, Georg Wolff, Frank G.A. Faas, Roman I. Koning, Thomas H. Sharp, Montserrat Bárcena and Abraham J. Koster

Cell and Chemical Biology/Electron Microscopy, Leiden University Medical Center, Leiden, The Netherlands.

In correlative light and electron microscopy (CLEM), both imaging modalities are often combined to study cellular processes. Fluorescence light microscopy (FM) enables imaging of dynamic events in relatively large fields of view and utilizes a wide range of available fluorescent markers, while electron microscopy (EM) can reveal structural details and macromolecular arrangements in their cellular context in relatively narrow fields of view at nm-scale resolution [1]. We will present several workflows for CLEM that we developed and applied, including novel instrumentation and imaging protocols.

For cell biology studies the use of EM often entails specimen preparation methods that incorporate dehydration, resin-embedding and metal staining. These methods can provide a wealth of information on the cellular architecture, especially when combined with high pressure freezing and freeze substitution. The 3D morphology of cell systems and tissue can be unraveled with nm-scale pixel size using electron tomography on sections several hundred nm thick. Larger volumes can be imaged with serial block face scanning EM (SEM). The flexibility in sample preparation procedures allows for the optimization of workflows that preserve and enhance the specific features of interest.

To improve our understanding of the biogenesis and dynamics of virus replication organelles we combined confocal live-cell imaging using a split-GFP fluorescent system with serial section block face SEM to provide sufficient temporal and spatial resolution in a whole-cell setting [2]. For applications where precise relocation accuracy is important, we developed a novel approach in which we show that the light-microscopy imaging of uranyl-stained and freeze-substituted sections under cryogenic conditions provides a means to accurately overlay the fluorescence with the transmission electron microscopy images without relying on fiducial markers [3].

At the molecular level, however, the fidelity of interpretation of conventionally prepared biological specimens is limited because of structural perturbations related to the normal fixation and staining steps. To capture macromolecular complexes that allow for interpretation at the molecular level, the specimens need to be preserved in a near-native state, that is by cryo-immobilization. In addition, to image inherently flexible complexes in the context of the cell the specimen needs to be sufficiently thin (less than $\sim 300 \mathrm{~nm}$ ) to facilitate cryo electron tomography. Recent technological improvements of image detectors and contrast-enhancing phase plates for transmission EM (TEM) have improved the contrast and signal-to-noise ratio of cryo EM datasets significantly. The ability to image macromolecular arrangements particularly when combined with sub-tomogram averaging provides to improve the features that are resolved, a powerful tool to understand molecular mechanisms.

Using cryo CLEM we targeted membrane structures specifically stained with fluorescent dye within whole bacteria. Subsequently, these membrane arrangements were imaged in 3D using cryo electron tomography. By imaging a large number of instances, a biological model of these membrane 
reorganization could be postulated. [4]. In a series of studies all related to a better understanding of the first activation steps of our innate immune system, we mimicked the membrane surface of a pathogen by liposomes and used this as a model system that, due to its thinness, produces higher resolution data [5]. By addition of antibodies and the molecules involved in this complement system cascade we aimed to dissect the process in molecular detail. For these electron tomography studies, the use of direct electron detectors and a phase plate was instrumental [6].

To target molecular arrangements in the context of a cell, sufficiently thin sections of prepared material are needed. We will show ongoing work where we combine focused ion beam milling with electron tomography. Also, we will discuss ongoing technological developments in our group that are aimed at realizing a workflow where cryo-light microscopy is used for navigation and super-resolution light microscopy is used to identify labeled structures in frozen-hydrated specimens [7].

\section{References:}

[1] SC Howes, RI Koning and AJ Koster, Curr Opin Microbiol 43 (2018), p. 132.

[2] HM van der Schaar, CE Melia et al, .mSphere 1 (2016), p. 104.

[3] MW Tuijtel et al, Sci Rep 7 (2017), p. 10442.

[4] K Celler et al, Nat Commun 7 (2016), p. 11836.

[5] TH Sharp et al, J Struct Biol 192 (2017), p. 155.

[6] D Ugurlar et al, Science 356 (2018), p. 794.

[7] The author acknowledges support by the Netherlands Organization (NWO) for Scientific Research (NWO-MEERVOUD grant 863.10.003), the Stichting Technologische Wetenschappen (STW) grants STW12713 and STW13711, the European Union through Horizon 2020 Programme iNext 653706, and from the Netherlands Centre for Electron Nanoscopy (NeCEN).

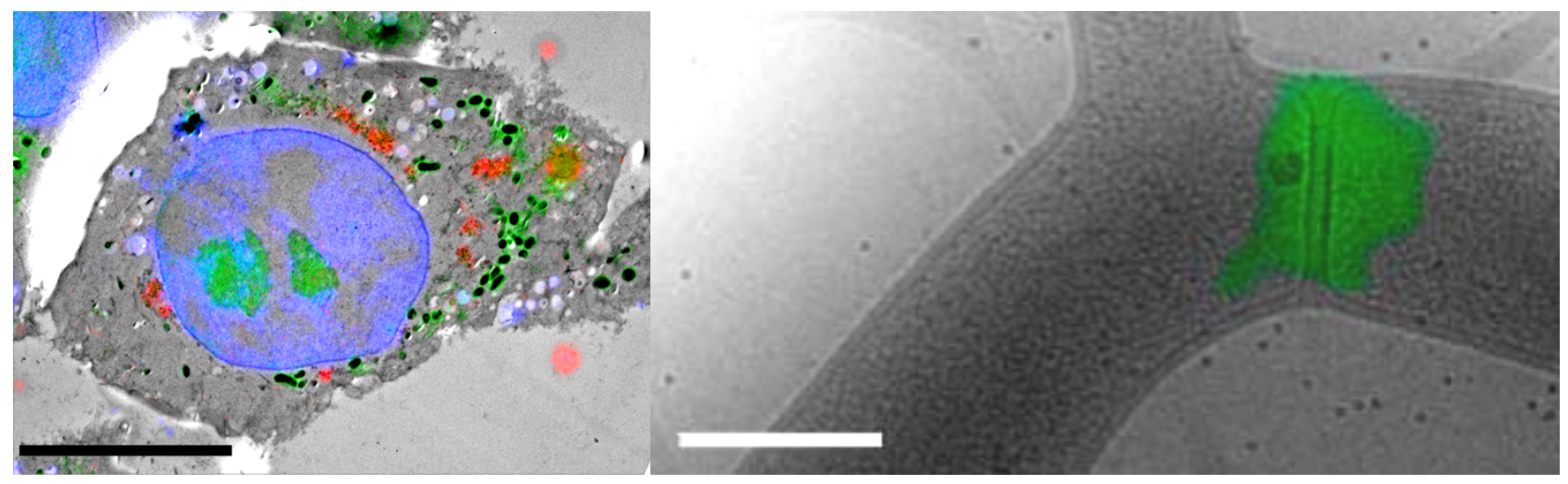

Figure 1. A. Overlay of three-color cryo fluorescence light microscopy images with the corresponding TEM image of a high-pressure-frozen freeze-substituted, and uranyl acetate stained section used to localize equine arteritis virus--induced replication organelles in monkey kidney cells (red). DAPI shown in blue. Scale bar $10 \mu \mathrm{m}$. Adapted from [4].B. Overlay of a cryo-fluorescence light microscopy image (green) with the corresponding cryo TEM image of a vegetative hyphae of Streptomyces albus stained with vancomycin-BODIPY dye highlighting the sites of septa within the bacterium. Scale bar $500 \mathrm{~nm}$. Adapted from [5]. 